ANNALS OF

CLINICAL

NEUROPHYSIOLOGY

\title{
Spectrum of nitrous oxide intoxication related neurological disorders in Korea: a case series and literature review
}

\author{
Jungsoo Lee ${ }^{1 *}$, Yangmi Park ${ }^{2^{*}}$, Hyunkee Kim ${ }^{1}$, Nakhoon Kim¹ ${ }^{1}$ Wonjae Sung ${ }^{1}$, Sanggon Lee ${ }^{1}$, \\ Jinseok Park ${ }^{1}$ \\ 'Department of Neurology, Hanyang Univeresity College of Medicine, Seoul, Korea \\ ${ }^{2}$ Department of Neurology, Seoul Metropolitan Government Boramae Medical Center, Seoul National University \\ College of Medicine, Seoul, Korea
}

Received: June 14, 2021

Revised: July 9, 2001

Accepted: July 29, 2001

\section{Correspondence to}

\section{Jinseok Park}

Department of Neurology, Hanyang University Hospital, 222-1 Wangsimni-ro, Seongdong-gu, Seoul 04763, Korea

Tel: +82-2-2290-8367

Fax: +82-2-2296-8370

E-mail:jinspark@hanyang.ac.kr

*These authors contributed equally.

ORCID

\section{Jungsoo Lee}

https://orcid.org/0000-0001-8390-6059

Yangmi Park

https://orcid.org/0000-0002-8765-9988

Hyunkee Kim

https://orcid.org/0000-0003-3824-6930

Nakhoon Kim

https://orcid.org/0000-0001-9002-9904

Wonjae Sung

https://orcid.org/0000-0002-4637-5890

\section{Sanggon Lee}

https://orcid.org/0000-0003-0622-1210

Jinseok Park

https://orcid.org/0000-0001-6581-583

Background: Nitrous oxide $\left(\mathrm{N}_{2} \mathrm{O}\right)$ is used in surgery and dentistry for its anesthetic and analgesic effects. However, neurological and psychiatric manifestations of $\mathrm{N}_{2} \mathrm{O}$ abuse have been increasingly reported among Korean adults. The aim of this study was to demonstrate laboratory findings of $\mathrm{N}_{2} \mathrm{O}$ abuse in Korean patients.

Methods: Patients diagnosed with $\mathrm{N}_{2} \mathrm{O}$-induced neuropathy or myelopathy from August 2018 to December 2019 were enrolled. Their clinical presentations and laboratory and imaging findings were analyzed.

Results: Sensory changes and limb weakness were present in nine of the enrolled patients. The laboratory findings revealed that seven patients had high homocysteine levels and five had high methylmalonic acid levels in their blood. Nerve conductions studies indicated that axonal neuropathy was present in four cases and longer F-wave and Hoffman's-reflex latencies were present in two cases. Signal changes in cervical spine imaging occurred in five patients, while two had normal results.

Conclusions: Chronic $\mathrm{N}_{2} \mathrm{O}$ abuse can cause neurological damage or psychiatric problems. Because $\mathrm{N}_{2} \mathrm{O}$ is illegal for recreational use in Korea, patients tend to hide their history of use. Even though the spinal imaging results were normal, clinicians should consider the possibility of $\mathrm{N}_{2} \mathrm{O}$ use, and further electrophysiological tests should be applied for precise evaluations.

key words: Nitrous oxide; Neuropathy; Spinal cord; Subacute combined degeneration; Homocysteine

This is an Open Acess article distributed under the terms of the Creative Commons Attribution Non-Commercial License /http:// creativecommons.org/licenses/by-nc/4.0) which permits unrestricted non-commercial use, distribution, and reproduction in any medium, provided the original work is properly cited. 


\section{INTRODUCTION}

Nitrous oxide $\left(\mathrm{N}_{2} \mathrm{O}\right)$ is a colorless and nonflammable gas that is used in surgery and dentistry due to its anesthetic effects. $\mathrm{N}_{2} \mathrm{O}$ has recently been misused for recreational purposes due to its euphoric effects when inhaled. The recreational use of $\mathrm{N}_{2} \mathrm{O}$ is currently prohibited in Korea due to its negative clinical effects, such as inducing vitamin $\mathrm{B}_{12}$ deficiency $^{1}$ or mechanisms that lead to thrombosis. ${ }^{2}$ However, $\mathrm{N}_{2} \mathrm{O}$ is still available in whipped-cream canisters or small bulbs that are predominantly used for recreation by young people. ${ }^{3}$ Reported cases of neurological and psychiatric manifestations of $\mathrm{N}_{2} \mathrm{O}$ abuse have recently increased among adults in Korea. ${ }^{4}$

The neurological complications associated with $\mathrm{N}_{2} \mathrm{O}$ abuse usually manifest as subacute combined degeneration (SCD) of the spinal cord. $\mathrm{N}_{2} \mathrm{O}$ deactivates the enzyme methionine synthase by inactivating methylcobalamin and impairing the methylation of myelin sheath proteins, leading to spinal cord degeneration and myelopathy. ${ }^{4}$ These processes can also cause peripheral nervous polyneuropathies. Several clinicians in Korea have reported various clinical symptoms and abnormal findings in tests after $\mathrm{N}_{2} \mathrm{O}$ inhalation, such as in electrophysiological examinations and magnetic resonance imaging (MRI). ${ }^{5}$ Additionally, vitamin $B_{12}$ deficiency tends to the induction of hyperhomocysteinemia, which in turn induces thrombosis. ${ }^{2}$ There are numerous previously reported cases of hyperhomocysteinemia inducing deep vein thrombosis or pulmonary thromboembolism (PTE). ${ }^{2,6}$

The use of illegal drugs such as cocaine, heroin, methamphetamine, and cannabis is reportedly lower in Korean than in other countries. ${ }^{7}$ Illegal drug usage is therefore considered by Korean clinicians less when they are determining symptom etiology. Since it is rare for patients to report their $\mathrm{N}_{2} \mathrm{O}$ use, diagnosing patients with atypical clinical manifestations is also difficult. A good understanding of the clinical manifestations of $\mathrm{N}_{2} \mathrm{O}$ usage is therefore important. Here we report nine patients with neurological manifestations and abnormal laboratory findings following $\mathrm{N}_{2} \mathrm{O}$ abuse.

\section{MATERIALS AND METHODS}

\section{Patient sample and clinical measurements}

We retrospectively analyzed clinical data from nine patients diagnosed with $\mathrm{N}_{2} \mathrm{O}$-induced neuropathy or myelopathy. This hospital-based case-series study investigated patients who presented at the Hanyang University Hospital (a tertiary referral medical center) in Seoul, Korea from August 2018 to December 2019. All patients underwent routine laboratory tests including a complete blood count, coagulation test, electrolyte test, and routine urine analysis. Considering the effects of $\mathrm{N}_{2} \mathrm{O}$ on vitamin $\mathrm{B}_{12}$ metabolism, serum methylmalonic acid (MMA), homocysteine, and vitamin $B_{12}$ levels were also examined. Electrophysiological tests such as a nerve conduction study (NCS) or electromyography were applied to eight patients. Spinal cord MRI, computed tomography (CT) scan with contrast enhancement, and chest CT angiography had been performed on seven, one, and one patient, respectively. Neurological examinations were performed, with the motor power of all four limbs measured according to the Medical Research Council grading system. Ethical approval was obtained for this study from the Institutional Review Board of the Hanyang University Hospital (IRB No. 2020-03-008).

\section{Electrophysiological examinations}

Standard motor and antidromic sensory NCSs were performed bilaterally on four motor nerves (median, ulnar, posterior tibial, and fibular) and three sensory nerves (median, ulnar, and sural) of eight patients. This analysis evaluated the terminal latency, compound muscle action potential amplitude (CMAP), and conduction velocity of each nerve. We defined abnormal results as changes of at least 20\% from the lower conduction velocity and CMAP limits, and from the upper terminal latency limit. Demyelinating NCS patterns were defined according to the guidelines from the American Association of Neuromuscular and Electrodiagnostic Medicine. ${ }^{8}$ F-wave latency was measured after supramaximal motor nerve stimulation, which identified ten F-waves. Hoffmann's reflex (H-reflex) was recorded from the soleus when stimulating the tibial nerve.

\section{Literature review}

Literature searches were performed of the MEDLINE and PubMed databases using the search terms "neuropathy $\mathrm{N}_{2} \mathrm{O}$ " and/or "myelopathy $\mathrm{N}_{2} \mathrm{O}$ " and including case reports published between 2006 and 2019 in South Korea. The reference lists of these reports were considered secondary 
Table 1. Clinical features and laboratory findings of nine patients with neurological effects from $\left(\mathrm{N}_{2} \mathrm{O}\right)$ intoxication

\begin{tabular}{|c|c|c|c|c|c|c|c|c|c|}
\hline Pt no. & Age & Sex & Duration $^{a}$ & Initial symptoms & Neurological & \multicolumn{2}{|c|}{ Laboratory data } & Spinal cord MRI & Other \\
\hline 1 & 28 & M & 3 weeks & $\begin{array}{l}\text { Weakness in both } \\
\text { limbs } \\
\text { Paresthesia in both } \\
\text { legs } \\
\text { (proximal } \rightarrow \text { distal) }\end{array}$ & $\begin{array}{l}\text { Quadriparesis (grade IV) } \\
\text { Hypesthesia below C5 } \\
\text { Tandem gait (-), Rom- } \\
\text { berg's sign (+) }\end{array}$ & $\begin{array}{l}\text { Hb } 12.2^{b}, \text { MCV } \\
100.3^{b} \\
\text { D-dimer } 1.83^{b} \\
\text { aPTT } 31\end{array}$ & $\begin{array}{l}\text { Vit } B_{12} 382.2 \\
\text { HCY } 56.7^{b} \\
\text { MMA } 12.27^{b}\end{array}$ & $\begin{array}{l}\text { T2-weighted hy- } \\
\text { perintensity at the } \\
\text { posterior column } \\
\text { (C2-C5) }\end{array}$ & $\begin{array}{l}\text { Pulmonary } \\
\text { artery } \\
\text { thromboem- } \\
\text { bolism } \\
\text { on chest CT }\end{array}$ \\
\hline 2 & 27 & $\mathrm{~F}$ & 3 weeks & $\begin{array}{l}\text { Gait disturbance } \\
\text { Acroparesthesia } \\
\text { Dyspepsia }\end{array}$ & $\begin{array}{c}\text { Decreased dorsiflexion } \\
\text { (grade IV) } \\
\text { Stocking-glove hypes- } \\
\text { thesia } \\
\text { Tandem gait (-), Rom- } \\
\text { berg's sign (+) } \\
\text { Dysmetria }\end{array}$ & $\begin{array}{l}\mathrm{Hb} 13.2, \mathrm{MCV} \\
103.2^{\mathrm{b}} \\
\text { D-dimer } 0.09 \\
\text { aPTT } 38\end{array}$ & $\begin{array}{l}\text { Vit } B_{12} 267.7 \\
\text { HCY } 45.4^{b} \\
\text { MMA } 9.44^{b}\end{array}$ & $\begin{array}{l}\text { T2-weighted hy- } \\
\text { perintensity at the } \\
\text { posterior column } \\
\text { (upper cervical } \\
\text { cord) }\end{array}$ & \\
\hline 3 & 24 & M & 16 weeks & $\begin{array}{l}\text { Weakness in both } \\
\text { leg } \\
\text { Numbness in both } \\
\text { legs }\end{array}$ & $\begin{array}{l}\text { Decreased dorsiflexion } \\
\text { (grade IV) } \\
\text { Hypesthesia in both legs } \\
\text { Tandem gait (-), Rom- } \\
\text { berg's sign (+) }\end{array}$ & $\begin{array}{c}\text { Hb 14.7 MCV } \\
97.5 \\
\text { D-dimer } 0.20 \\
\text { aPTT } 33\end{array}$ & $\begin{array}{c}\text { Vit } B_{12} \\
>2,000 \\
\text { HCY } 10.7^{b} \\
\text { MMA } 1.00\end{array}$ & Not checked & \\
\hline 4 & 23 & $\mathrm{~F}$ & 4 weeks & $\begin{array}{l}\text { Weakness in both } \\
\qquad \text { limbs } \\
\text { (distal } \rightarrow \text { proximal) } \\
\text { Acroparesthesia }\end{array}$ & $\begin{array}{l}\text { Decreased dorsiflexion } \\
\text { (grade III) } \\
\text { Quadriparesis (grade IV) } \\
\text { Stocking-glove hypes- } \\
\text { thesia } \\
\text { Hypoactive DTR } \\
\text { Decreased anal tone } \\
\text { Tandem gait (-), Rom- } \\
\text { berg's sign (+) }\end{array}$ & $\begin{array}{l}\mathrm{Hb} 11.5^{\mathrm{b}}, \mathrm{MCV} \\
106.1^{\mathrm{b}} \\
\text { D-dimer } 0.52^{\mathrm{b}} \\
\text { aPTT } 27\end{array}$ & $\begin{array}{l}\text { Vit } B_{12} \\
>2,000 \\
\text { HCY } 25.0 \\
\text { MMA } 2.63\end{array}$ & $\begin{array}{l}\text { T2-weighted hy- } \\
\text { perintensity at the } \\
\text { posterior column } \\
\text { (C2-C6) }\end{array}$ & \\
\hline 5 & 28 & M & 2 weeks & $\begin{array}{l}\text { Weakness in both } \\
\text { ankles } \\
\text { Acroparesthesia }\end{array}$ & $\begin{array}{l}\text { Decreased dorsiflexion } \\
\text { (grade IV) } \\
\text { Stocking-glove hypes- } \\
\text { thesia } \\
\text { Hypoactive DTR } \\
\text { Tandem gait (-) }\end{array}$ & $\begin{array}{l}\text { Hb 16.9, MCV } \\
\text { 100.6 } \\
\text { D-dimer not } \\
\text { checked } \\
\text { aPTT } 36\end{array}$ & $\begin{array}{l}\text { Vit } B_{12} 201.6 \\
\text { HCY } 45.6^{b} \\
\text { MMA not } \\
\text { checked }\end{array}$ & No signal change & \\
\hline 6 & 24 & M & Unknown & $\begin{array}{c}\text { Weakness in both } \\
\text { legs } \\
\text { (distal } \rightarrow \text { proximal) } \\
\text { Paresthesia in in both } \\
\text { legs }\end{array}$ & $\begin{array}{c}\text { Quadriparesis } \\
\text { (proximal, grade IV; } \\
\text { distal, grade II) } \\
\text { Stocking-glove hypes- } \\
\text { thesia } \\
\text { Areflexia } \\
\text { Tandem gait (-), Rom- } \\
\text { berg's sign (+) }\end{array}$ & $\begin{array}{c}\text { Hb 16.9, MCV } \\
\text { 100.6 } \\
\text { D-dimer not } \\
\text { checked } \\
\text { aPTT } 37\end{array}$ & $\begin{array}{l}\text { Vit } B_{12} 414.5 \\
\text { HCY } 45.5^{b} \\
\text { MMA } 5.86^{b}\end{array}$ & Not checked & \\
\hline 7 & 26 & $\mathrm{~F}$ & 2 weeks & $\begin{array}{c}\text { Numbness in both } \\
\text { legs } \\
\text { (distal } \rightarrow \text { proximal) }\end{array}$ & $\begin{array}{l}\text { Vibration loss in both } \\
\text { legs } \\
\text { Hypoactive DTR } \\
\text { Tandem gait (-), Rom- } \\
\text { berg's sign (+) }\end{array}$ & $\begin{array}{c}\mathrm{Hb} 11.3^{\mathrm{b}}, \mathrm{MCV} \\
102.5^{\mathrm{b}} \\
\text { aPTT } 27 \\
\text { D-dimer } 0.14\end{array}$ & $\begin{array}{l}\text { Vit } B_{12} 162.1^{b} \\
\text { HCY } 49.1^{b} \\
\text { MMA } 4.87^{b}\end{array}$ & No signal change & \\
\hline
\end{tabular}


Table 1. Continued

\begin{tabular}{|c|c|c|c|c|c|c|c|c|c|}
\hline Pt no. & $\begin{array}{c}\text { Age } \\
\text { (years) }\end{array}$ & Sex & Duration $^{\mathrm{a}}$ & Initial symptoms & $\begin{array}{l}\text { Neurological } \\
\text { examination }\end{array}$ & \multicolumn{2}{|c|}{ Laboratory data } & Spinal cord MRI & $\begin{array}{l}\text { Other } \\
\text { findings }\end{array}$ \\
\hline 8 & 22 & $\mathrm{~F}$ & 12 weeks & Acroparesthesia & $\begin{array}{l}\text { Stocking-glove hypes- } \\
\text { thesia } \\
\text { Tandem gait (-), Rom- } \\
\text { berg's sign (+) }\end{array}$ & $\begin{array}{c}\text { Hb 13.5 MCV } \\
101.6^{\mathrm{b}} \\
\text { D-dimer } 0.08 \\
\text { aPTT } 33\end{array}$ & $\begin{array}{c}\text { Vit } B_{12} 1,456 \\
\text { HCY } 11.2 \\
\text { MMA } 3.32\end{array}$ & $\begin{array}{l}\text { T2-weighted hy- } \\
\text { perintensity at the } \\
\text { posterior column } \\
\text { (C2 and C3) }\end{array}$ & \\
\hline 9 & 35 & $\mathrm{~F}$ & 12 weeks & $\begin{array}{l}\text { Weakness in both } \\
\text { legs } \\
\text { Paresthesia in both } \\
\text { legs }\end{array}$ & $\begin{array}{c}\text { Paraparesis } \\
\text { Vibration loss in both } \\
\text { legs } \\
\text { Hyperactive DTR, } \\
\text { Babinski sign (+) }\end{array}$ & $\begin{array}{c}\text { Hb } 11 \text { MCV } 107.1 \\
\text { D-dimer } 1.1 \\
\text { aPTT } 31\end{array}$ & $\begin{array}{l}\text { Vit } B_{12} 222.1 \\
\text { HCY } 76.4 \\
\text { MMA not } \\
\text { checked }\end{array}$ & $\begin{array}{c}\text { T2-weighted } \\
\text { hyperintensity at } \\
\text { posterior, anterior, } \\
\text { and lateral column } \\
\text { (C7 to conus } \\
\text { medullaris) }\end{array}$ & $\begin{array}{c}\text { Chest CT } \\
\text { angiography } \\
\text { showed no } \\
\text { specific lesions }\end{array}$ \\
\hline
\end{tabular}

Pt, patient; MRI, magnetic resonance imaging; $\mathrm{M}$, male; $\mathrm{Hb}$, hemoglobin (normal range = 12-18 g/dL); MCV, mean corpuscular volume (normal range = 80-99 fL); D-dimer (normal range = 0-0.24 mg/L); aPTT, activated partial thromboplastin time (normal range $=25-39$ seconds); Vit $B_{12}$, vitamin $B_{12}$ (normal range $=197-771 \mathrm{pg} / \mathrm{mL}$ ); HCY, homocysteine (normal range $=0-15 \mu \mathrm{mol} / \mathrm{L}$ ); MMA, methylmalonic acid (normal range $=0.01-3.76 \mathrm{mg} / \mathrm{g}$ ); $\mathrm{CT}$, computed tomography; F, female; DTR, deep tendon reflex.

anterval between time of last $\mathrm{N}_{2} \mathrm{O}$ inhalation and symptom onset as reported by the patient; ${ }^{b}$ Abnormal laboratory findings.

sources. Cases without postmortem analyses or NCS results were excluded.

\section{RESULTS}

Nine patients aged between 23 and 35 years visited our hospital from August 2017 to December 2019 (Table 1). All patients had a history of $\mathrm{N}_{2} \mathrm{O}$ inhalation during the 6 months prior to admission. The interval between the last $\mathrm{N}_{2} \mathrm{O}$ inhalation and symptom onset varied from 2 weeks to 4 months, while the interval between symptom onset and hospital visit varied from 3 days to 1 month, with the majority of patients visiting 1 week after symptom onset. Five patients were female. Sensory change was the initial symptom of all patients. These sensory symptoms varied between patients and included an ascending tingling sensation in both legs (patient no. 1), numbness in both legs (patient no. 3 and 7), and acroparesthesia (patient no. 2, 4, 5, 8, and 9). Five patients experienced muscle weakness, three of which only had lower limb weakness (patient no. 3, 6, and 9), and two had both upper and lower limb weakness (patient no. 1 and 4). Muscle weakness only in the ankles was found in patient no. 5. One patient had dyspepsia. None of the patients were vegetarians or had previously received gastrointestinal surgery.
Neurological examinations indicated that seven of the nine patients had limb paresis. Three had mild weakness in ankle dorsiflexion and the other four had quadriparesis. Sensory changes were detected in patient no. 1, while patient no. 7 and 9 had a loss of vibration sensation in both legs. Among the other patients, six had stocking-glove hypesthesia (patient no. 2, 4, 5, 6, 8, and 9). Hypoactivity of the deep tendon reflex (DTR) occurred in four cases (patient no. 4, 5, 6, and 7), four had normoactive DTR, and one had hyperactive DTR (patient no. 9). Patient no. 2 had dysmetria of the upper limbs and all patients except no. 5 showed abnormal results in the Romberg test. All patients had an impaired tandem gait. Digital rectal examinations indicated that patient no. 4 had decreased anal tone.

Laboratory findings revealed low hemoglobin levels and large mean corpuscular volumes in four and six patients, respectively. Only one patient had a low serum vitamin $B_{12}$ level (patient no. 7). Elevated serum homocysteine levels were indicated in seven cases (patient no. 1, 2, 3, 5, 6, 7, and 9), and five patients had high blood MMA levels.

NCSs were performed on eight patients (Table 2). Among them, four had axonal neuropathies (patient nos. 3, 4, 5, and 6) and one had longer $\mathrm{F}$-wave and $\mathrm{H}$-reflex latencies in both lower limbs (patient no. 7). Seven patients had a decreased sural nerve conduction velocity or longer H-reflex latency. 
Most patients had more abnormalities in the lower limbs than in the upper limbs, distal limb regions were affected more than were proximal regions, and sensory nerves were involved more frequently than were motor nerves. Demyelination was not observed in any patient. The reference values and raw data of patients' nerve conduction study are shown respectively on Supplementary Table 1, 2.

Seven patients received spinal MRI, five of whom had signal changes in the dorsal column of the cervical spine, as indicated in T2-weighted images (Fig. 1). Patient no. 9 had concurrent hyperintensity lesions in the anterior, lateral, and posterior spinal column (Fig. 2). Two patients (patient no. 5 and 7) showed normal results in spinal MRI.

All patients received hydroxocobalamin as a vitamin $B_{12}$ supplement. Three patients received additional steroid therapy. Intravenous immunoglobulin G was administered to one patient (patient no. 5) due to their observed clinical features being similar to those associated with early Guillain-Barré syndrome. Patient no.1 suffered from $\mathrm{N}_{2} \mathrm{O}$ addiction and depression, so they received psychiatric consulting.

A literature search identified five articles describing SCD attributed to recreational $\mathrm{N}_{2} \mathrm{O}$ abuse, including three male

Table 2. Abnormal findings in NCSs of nine patients

\begin{tabular}{|c|c|c|c|c|c|c|c|c|c|}
\hline Pt no. & $\begin{array}{l}\text { Median } \\
\text { nerve } \\
\text { (motor) }\end{array}$ & $\begin{array}{c}\text { Median } \\
\text { nerve } \\
\text { (sensory) }\end{array}$ & $\begin{array}{l}\text { Ulnar nerve } \\
\text { (motor) }\end{array}$ & $\begin{array}{l}\text { Ulnar nerve } \\
\text { (sensory) }\end{array}$ & $\begin{array}{l}\text { Posterior tibial } \\
\text { nerve (motor) }\end{array}$ & $\begin{array}{l}\text { Fibular nerve } \\
\text { (motor) }\end{array}$ & $\begin{array}{l}\text { Sural nerve } \\
\text { (sensory) }\end{array}$ & $\begin{array}{l}\text { H-reflex } \\
\text { latency }\end{array}$ & Conclusion \\
\hline 1 & & & $\begin{array}{c}\text { F-wave } \\
\text { latency } \uparrow \\
\text { (Rt) }\end{array}$ & & & CMAP $\downarrow$ (both) & $\mathrm{CV} \downarrow$ (both) & & $\begin{array}{l}\text { Sensorimotor } \\
\text { polyneuropa- } \\
\text { thy in leg }\end{array}$ \\
\hline 2 & & & & & $\begin{array}{c}\text { F-wave latency } \\
\uparrow \text { (both) }\end{array}$ & $\begin{array}{c}\text { F-wave latency } \\
\uparrow \text { (both) }\end{array}$ & $\mathrm{CV} \downarrow$ (both) & $\begin{array}{c}\text { H-reflex } \\
\text { latency } \uparrow \\
\text { (both) }\end{array}$ & $\begin{array}{l}\text { Sensorimotor } \\
\text { polyneuropa- } \\
\text { thy }\end{array}$ \\
\hline 3 & & $\mathrm{CV} \downarrow$ (both) & & $C V \downarrow(L t)$ & $\begin{array}{c}\text { F-wave latency } \\
\uparrow(\mathrm{Lt})\end{array}$ & $\begin{array}{c}\text { CMAP } \downarrow \text { (both) } \\
\text { CV } \downarrow \text { (Lt) } \\
\text { F-wave latency } \\
\uparrow(\mathrm{Rt})\end{array}$ & CV $\downarrow$ (both) & $\begin{array}{l}\text { H-reflex } \\
\text { latency } \uparrow \\
\text { (both) }\end{array}$ & AMSAN \\
\hline 4 & & $C V \downarrow(L t)$ & & $\mathrm{CV} \downarrow$ (both) & $\begin{array}{c}\mathrm{CV} \downarrow \text { (Rt) } \\
\text { F-wave latency } \\
\uparrow \text { (both) }\end{array}$ & $\begin{array}{c}\text { CMAP } \downarrow \text { (both) } \\
C \vee \downarrow \text { (both) } \\
\text { F-wave latency } \\
\uparrow \text { (both) }\end{array}$ & $C \vee \downarrow$ (both) & $\begin{array}{l}\text { H-reflex } \\
\text { latency } \uparrow \\
\text { (both) }\end{array}$ & AMSAN \\
\hline 5 & & CV $\downarrow$ (both) & $\begin{array}{l}C V \downarrow(L t) \\
T L \uparrow(R t)\end{array}$ & CV $\downarrow$ (both) & $\begin{array}{c}\text { CMAP } \downarrow \text { (both) } \\
\mathrm{TL} \uparrow \text { (both) } \\
\mathrm{CV} \downarrow \text { (Rt) }\end{array}$ & $\begin{array}{c}\text { No CMAP } \\
\text { (both) }\end{array}$ & $\mathrm{CV} \downarrow(\mathrm{Rt})$ & $\begin{array}{c}\text { H-reflex } \\
\text { latency } \uparrow \\
\text { (both) }\end{array}$ & AMSAN \\
\hline 6 & $\begin{array}{l}\text { CMAP } \downarrow \text { (Lt) } \\
\text { CV } \downarrow \text { (both) }\end{array}$ & $\mathrm{CV} \downarrow$ (both) & $\mathrm{TL} \uparrow(\mathrm{Rt})$ & $\mathrm{CV} \downarrow$ (both) & No CMAP (both) & $\begin{array}{c}\text { No CMAP } \\
\text { (both) }\end{array}$ & $C V \downarrow(L t)$ & $\begin{array}{l}\text { No H-reflex } \\
\text { (both) }\end{array}$ & AMSAN \\
\hline 7 & & & & & $\begin{array}{c}\text { F-wave latency } \\
\uparrow \text { (both) }\end{array}$ & $\begin{array}{c}\text { F-wave latency } \\
\uparrow \text { (both) }\end{array}$ & & $\begin{array}{c}\text { H-reflex } \\
\text { latency } \uparrow \\
\text { (both) }\end{array}$ & Normal \\
\hline 8 & & $C V \downarrow(R t)$ & & $\mathrm{CV} \downarrow$ (both) & & $\begin{array}{c}\text { CMAP } \downarrow(L t) \\
\text { F-wave latency } \\
\uparrow \text { (both) }\end{array}$ & CV $\downarrow$ (both) & $\begin{array}{l}\text { H-reflex } \\
\text { latency } \uparrow \\
\text { (both) }\end{array}$ & $\begin{array}{l}\text { Sensory domi- } \\
\text { nant polyneu- } \\
\text { ropathy }\end{array}$ \\
\hline 9 & Not checked & & & & & & & & \\
\hline
\end{tabular}

Undocumented findings were normal.

NCSS, nerve conduction studies; H-reflex, Hoffman's reflex; Rt, right; CMAP, compound motor action potential amplitude; CV, conduction velocity; Lt, left; AMSAN, acute motor-sensory axonal neuropathy; $\mathrm{TL}$, terminal latency. 
and seven female cases. All of these cases had gait disturbances or sensory changes as initial symptoms accompanied by signal changes in cervical MRI. NCSs indicated neuropathic patterns or early-stage polyneuropathy. One patient presented with incidental PTE and lung infarction. Table 3 summarizes the details of the published cases. ${ }^{5,9,10}$

\section{DISCUSSION}

$\mathrm{N}_{2} \mathrm{O}$ is known to selectively oxidize folate and vitamin $\mathrm{B}_{12}$ rendering them inactive and unable to degrade homocysteine into methionine, which is required for normal myelin production. ${ }^{11}$ Active vitamin $B_{12}$ is also required to convert MMA into succinyl-CoA. Serum levels of both homocysteine
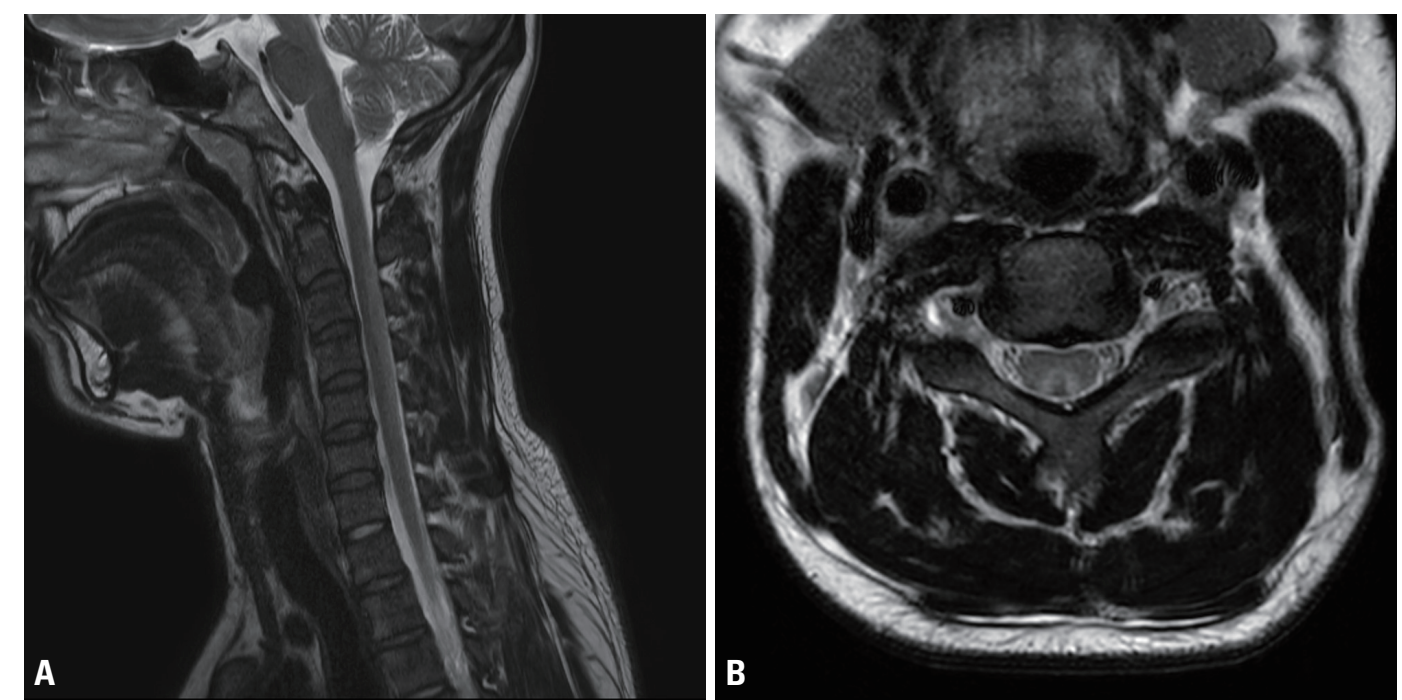

Fig. 1. T2-weighted cervical spinal magnetic resonance imaging (patient no. 1). (A) In the sagittal series, abnormal hyperintensities were observed in the dorsal cervical spinal cord (C2-C5) without enhancement. (B) In the axial series, high signal intensity (inverted-V sign) and cord swelling were observed.
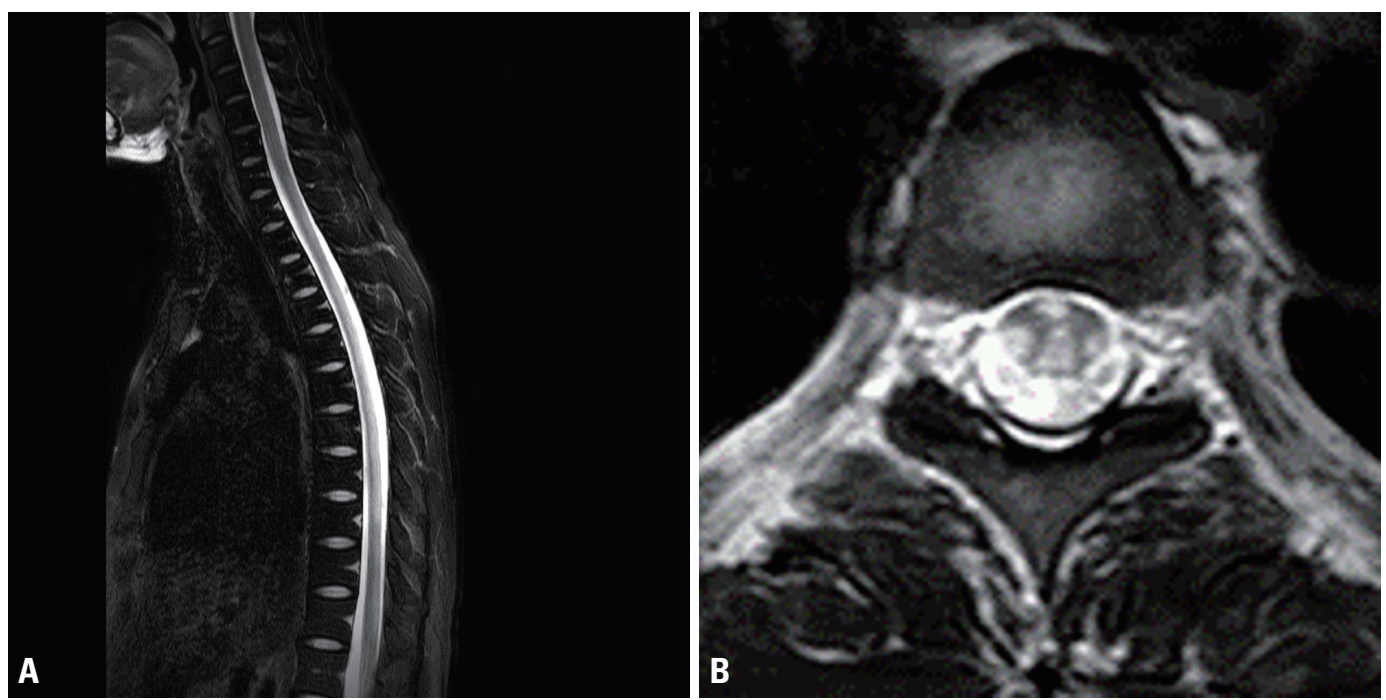

Fig. 2. T2-weighted spinal magnetic resonance imaging (patient no. 9). (A) T2-weighted sagittal image, indicating high signal intensities within the posterior spinal cord from C7 to the conus medullaris. (B) T2-weighted axial image indicating high signal intensities in the anterior, lateral, and posterior spinal column. 
Table 3. Published cases of nitrous oxide intoxication with neurological manifestations

\begin{tabular}{|c|c|c|c|c|c|c|c|c|}
\hline Year & Ref. & $\begin{array}{l}\operatorname{Age}^{\mathrm{a}} / \\
\operatorname{sex}\end{array}$ & Symptoms & $\begin{array}{c}\text { Neurological examina- } \\
\text { tion }\end{array}$ & $\begin{array}{l}\text { Laboratory find- } \\
\text { ings }\end{array}$ & MRI changes & NCS & Others \\
\hline 2019 & $\begin{array}{l}\text { Lee et } \\
\text { al. }^{10}\end{array}$ & $32 / F$ & Gait disturbance & $\begin{array}{c}\text { Romberg's sign (+), } \\
\text { sensory ataxia }\end{array}$ & $\begin{array}{l}\text { Low Vit } \mathrm{B}_{12} \\
\text { High homocysteine }\end{array}$ & $\begin{array}{l}\text { Posterior column } \\
\text { of } \mathrm{C} 1 \text { and } \mathrm{C} 2\end{array}$ & Not mentioned & \\
\hline \multirow[t]{4}{*}{2018} & \multirow[t]{4}{*}{$\begin{array}{l}\text { Kang et } \\
\text { al. }^{19}\end{array}$} & $? / F$ & $\begin{array}{l}\text { Progressive limb } \\
\text { paralysis }\end{array}$ & $\begin{array}{c}\text { Motor weakness in lower } \\
\text { extremities }\end{array}$ & $\begin{array}{c}\text { Increased MCV } \\
\text { Low Vit } \mathrm{B}_{12} \\
\text { High homocysteine }\end{array}$ & $\begin{array}{l}\text { Posterior column } \\
\text { of cervical cord }\end{array}$ & $\begin{array}{l}\text { Demyelinating } \\
\text { polyneuropathy }\end{array}$ & \\
\hline & & $? / F$ & $\begin{array}{l}\text { Progressive limb } \\
\text { paralysis, paresthe- } \\
\text { sia }\end{array}$ & $\begin{array}{c}\text { Romberg's sign }(+), \\
\text { motor weakness in lower } \\
\text { extremities, } \\
\text { hypesthesia to vibration, } \\
\text { position }\end{array}$ & $\begin{array}{c}\text { Increased MCV } \\
\text { Low Vit } \mathrm{B}_{12} \\
\text { High homocysteine }\end{array}$ & $\begin{array}{l}\text { Posterior column } \\
\text { of cervical cord }\end{array}$ & $\begin{array}{l}\text { Demyelinating } \\
\text { polyneuropathy }\end{array}$ & \\
\hline & & $? / F$ & $\begin{array}{l}\text { Progressive limb } \\
\text { paralysis, paresthe- } \\
\text { sia }\end{array}$ & $\begin{array}{l}\text { Romberg's sign (+), } \\
\text { motor weakness in lower } \\
\text { extremities, } \\
\text { hypesthesia to vibration, } \\
\text { position }\end{array}$ & $\begin{array}{c}\text { Increased MCV } \\
\text { Low Vit } \mathrm{B}_{12} \\
\text { High homocysteine }\end{array}$ & $\begin{array}{l}\text { Posterior column } \\
\text { of cervical cord }\end{array}$ & Normal & \\
\hline & & $? / F$ & $\begin{array}{l}\text { Progressive limb } \\
\text { paralysis, paresthe- } \\
\text { sia }\end{array}$ & $\begin{array}{c}\text { Motor weakness in lower } \\
\text { extremities, } \\
\text { paresthesia }\end{array}$ & $\begin{array}{l}\text { Macrocytic anemia } \\
\text { Low Vit } \mathrm{B}_{12} \\
\text { High homocysteine }\end{array}$ & $\begin{array}{l}\text { Posterior column } \\
\text { of cervical cord }\end{array}$ & $\begin{array}{l}\text { Axonal polyneu- } \\
\text { ropathy }\end{array}$ & $\begin{array}{l}\text { Incidental } \\
\text { PTE with lung } \\
\text { infarction }\end{array}$ \\
\hline \multirow[t]{2}{*}{2018} & \multirow[t]{2}{*}{$\begin{array}{l}\text { Kwon et } \\
\text { al. }^{18}\end{array}$} & $22 / F$ & $\begin{array}{l}\text { Gait disturbance, } \\
\text { progressive pares- } \\
\text { thesia in legs and } \\
\text { hands }\end{array}$ & $\begin{array}{l}\text { Paraparesis (MRC grade III) } \\
\text { Romberg's sign }(+) \text {, decom- } \\
\text { position, } \\
\text { hypesthesia to vibration, } \\
\text { tactile }\end{array}$ & $\begin{array}{c}\text { Increased MCV } \\
\text { Low Vit } B_{12}\end{array}$ & $\begin{array}{l}\text { Posterior column } \\
\text { of cervical cord }\end{array}$ & $\begin{array}{l}\text { Axonal motor } \\
\text { neuropathy }\end{array}$ & \\
\hline & & $33 / \mathrm{M}$ & $\begin{array}{l}\text { Gait disturbance, } \\
\text { progressive numb- } \\
\text { ness in legs }\end{array}$ & $\begin{array}{c}\text { Romberg's sign (+), sensory } \\
\text { ataxia, } \\
\text { hypesthesia to vibration, } \\
\text { position, hyporeflexia }\end{array}$ & $\begin{array}{l}\text { Increased MCV } \\
\text { Low Vit } B_{12}\end{array}$ & $\begin{array}{l}\text { Posterior column } \\
\text { of cervical cord }\end{array}$ & $\begin{array}{l}\text { Axonal motor } \\
\text { neuropathy }\end{array}$ & \\
\hline \multirow[t]{2}{*}{2018} & \multirow[t]{2}{*}{$\begin{array}{l}\text { Choi et } \\
\text { al. }^{9}\end{array}$} & $24 / M$ & $\begin{array}{l}\text { Gait disturbance, } \\
\text { paresthesia in all } \\
\text { limbs, } \\
\text { voiding difficulty }\end{array}$ & $\begin{array}{c}\text { Romberg's sign (+), gait } \\
\text { ataxia, } \\
\text { hypesthesia, paresthesia to } \\
\text { tactile, } \\
\text { hyperreflexia }\end{array}$ & $\begin{array}{l}\text { Increased MCV } \\
\text { High homocysteine }\end{array}$ & $\begin{array}{l}\text { Posterior column } \\
\text { of cervical cord }\end{array}$ & $\begin{array}{l}\text { Sensorimotor } \\
\text { polyneuropathy } \\
\text { in lower limbs }\end{array}$ & \\
\hline & & $22 / F$ & $\begin{array}{l}\text { Progressive leg } \\
\text { paralysis, voiding } \\
\text { difficulty, } \\
\text { paresthesia in legs }\end{array}$ & $\begin{array}{c}\text { Paraparesis (MRC grade II), } \\
\text { hypesthesia to tactile, } \\
\text { areflexia }\end{array}$ & $\begin{array}{l}\text { Low Vit } \mathrm{B}_{12} \\
\text { High homocysteine }\end{array}$ & $\mathrm{C} 2-\mathrm{C} 5$ & $\begin{array}{l}\text { Sensorimotor } \\
\text { polyneuropathy } \\
\text { in lower limbs }\end{array}$ & \\
\hline 2006 & $\begin{array}{l}\text { Kwoun } \\
\text { et al. }{ }^{5}\end{array}$ & 40/M & $\begin{array}{c}\text { Gait disturbance, } \\
\text { numbness in hands } \\
\text { and feet }\end{array}$ & $\begin{array}{l}\text { Hypesthesia to pain, vibra- } \\
\text { tion, position, } \\
\text { Romberg's sign (+), gait } \\
\text { ataxia, } \\
\text { hyperreflexia }\end{array}$ & $\begin{array}{l}\text { Microcytic hypo- } \\
\text { chromic anemia } \\
\text { Low Vit } \mathrm{B}_{12} \\
\text { High homocysteine }\end{array}$ & $\begin{array}{l}\text { Posterior column } \\
\text { and lateral col- } \\
\text { umn of cervical } \\
\text { cord }\end{array}$ & $\begin{array}{l}\text { Demyelinating } \\
\text { polyneuropathy }\end{array}$ & \\
\hline
\end{tabular}

Ref., reference number; MRI, magnetic resonance imaging; NCS, nerve conduction study; F, female; Vit $B_{12}$, vitamin $B_{12}$; MCV, mean corpuscular volume; PTE, pulmonary thromboembolism; MRC, Medical Research Council; M, male.

${ }^{a}$ All patients were aged $21-36$ years; ${ }^{b}$ Active movement against gravity. 
and MMA are elevated during vitamin $\mathrm{B}_{12}$ deficiency. Previous in vitro studies have demonstrated the cytotoxic effect of homocysteine on cortical astrocytes and that of MMA on primary neuronal cultures. ${ }^{12,13}$ This interference with vitamin $B_{12}$ metabolism could lead to demyelination in the central or peripheral nervous system and also to megaloblastic anemia. These pathophysiological mechanisms result in $\mathrm{N}_{2} \mathrm{O}$ intoxication being associated with clinical features such as numbness in the extremities, ataxia, and psychomotor symptoms (including impaired memory function and depression). ${ }^{14}$

There were marked variations in clinical symptoms, laboratory findings, electrophysiological findings, and MRI results associated with $\mathrm{N}_{2} \mathrm{O}$ usage among the cases examined in this study. Sensory nerve fibers are generally affected more frequently by toxins. ${ }^{15}$ Similar to other toxic neuropathies, we found that all of our patients experienced sensory changes in their distal limbs, and that these changes were correlated with NCS abnormalities. Motor weakness was observed in 50\% of cases, but motor NCS abnormalities were not found to be correlated with these symptoms. In two cases (patient no. 1 and 7), only F-wave and H-reflex latency changes were identified. These findings resembled the early clinical features of Guillain-Barré syndrome, but our results indicated no demyelinating patterns. Considering the relationships between $\mathrm{N}_{2} \mathrm{O}$, vitamin $\mathrm{B}_{12}$ deficiency, and hypomyelination, these axonal patterns detected through $\mathrm{NCSs}$ suggest that an alternative mechanism of $\mathrm{N}_{2} \mathrm{O}$ directly contributes to axonal damage. ${ }^{16}$

Most of the patients had high serum homocysteine or MMA levels despite normal vitamin $B_{12}$ levels. Due to the illegality of recreational $\mathrm{N}_{2} \mathrm{O}$ use in Korea, most patients with neurological symptoms were assumed to have ingested vitamin $B_{12}$ prior to visiting our hospital. A previous case report indicated that homocysteine and MMA levels could be normalized by vitamin $B_{12}$ treatment. ${ }^{17}$

MRI findings in previously reported cases of $\mathrm{N}_{2} \mathrm{O}$ intoxication related to SCD have frequently identified symmetric bilateral T2-weighted hyperintensity lesions at the posterior spinal cord. This specific finding is known as the "inverted-V sign." In the present case series, the MRI findings of about half of the patients were compatible with SCD. However, two patients who had no detected MRI signal changes had abnormal NCS results, which represents the first report of this phenomenon in Korea. One patient also had concurrent involvement in the anterior, lateral, and posterior spinal column.

There have been several reports of the deleterious effects of $\mathrm{N}_{2} \mathrm{O}$ abuse in Korea. Kwoun et al. ${ }^{5}$ reported the first case of myeloneuropathy following chronic $\mathrm{N}_{2} \mathrm{O}$ abuse in 2003. In that report, the patient had paresthesia in both distal limbs and ataxia. Spinal MRI indicated high signal intensities from segments C2 to C5 on a T2-weighted image. Furthermore, NCSs indicated a demyelinating neuropathic pattern. Serum vitamin $\mathrm{B}_{12}$ levels were low and those of homocysteine were high. Two other cases of SCD caused by $\mathrm{N}_{2} \mathrm{O}$ gas were reported by Kwon et al. ${ }^{18}$ in 2019. Both patients had sensory changes in their limbs and the inverted-V sign was visible in T2-weighted spinal MRI. NCSs indicated axonal motor polyneuropathy in that case. In all previous cases of $\mathrm{N}_{2} \mathrm{O}$ intoxication in Korea, spinal cord MRI indicated signal changes similar to those observed in SCD. Unlike previously reported cases, we found two patients who had abnormal NCS results despite having normal spinal MRI results. We therefore suggest that clinicians need to consider the possibility of $\mathrm{N}_{2} \mathrm{O}$ inhalation in patients whose NCSs produce axonal degeneration findings, even if signal changes are not observed in spinal cord MRI.

The present study has revealed that $\mathrm{N}_{2} \mathrm{O}$ abuse may be associated with a wide range of clinical features, including psychiatric problems such as addiction and depression, especially among adolescents and young adults. Due to the illegality of recreational $\mathrm{N}_{2} \mathrm{O}$ use in Korea, patients tend to hide their history of $\mathrm{N}_{2} \mathrm{O}$ use or inaccurately report symptom onset. Addressing this issue requires treatments for neurological deficits, the careful recording of the medical histories, and psychiatric support for patients.

Our study had several limitations. First, its retrospective design made it difficult to precisely evaluate and quantify the data analyzed for each patient. Second, this study included only nine cases from a single center, so it might not be representative of all patients experiencing $\mathrm{N}_{2} \mathrm{O}$ intoxication in Korea. Third, all patients had supplemented their vitamin $\mathrm{B}_{12}$ intake before the clinical evaluations, which makes it difficult to accurately interpret the results. Fourth, the patients tended to not reveal their history of $\mathrm{N}_{2} \mathrm{O}$ inhalation because it is currently illegal to use $\mathrm{N}_{2} \mathrm{O}$ gas for recreational purposes in Korea. It was therefore difficult to evaluate the correlations 
between clinical features and the exact amount and duration of $\mathrm{N}_{2} \mathrm{O}$ inhalation.

The clinical manifestations of $\mathrm{N}_{2} \mathrm{O}$ intoxication related to neurological disorders varied between the included patients, but had a general commonality. Several clinical indicators may include young adults with progressive subacute weakness and lower-extremity-dominant sensory changes. Increased serum MMA, homocysteine, axonal-type sensorimotor polyneuropathy patterns in electrophysiological tests, and predominant posterior column involvement observed in MRI may indicate the presence of $\mathrm{N}_{2} \mathrm{O}$ intoxication related to neurological disorders. Careful evaluations of the clinical history of $\mathrm{N}_{2} \mathrm{O}$ abuse are therefore warranted when these clinical findings are observed.

\section{Conflicts of Interest}

The authors have no conflicts to disclose.

\section{Acknowledgements}

This work was supported by the research fund of Hanyang University(HY-202100000001136).

\section{Supplementary Material}

Supplementary Materials can be found with this article online https://doi.org/10.14253/acn.2021.23.2.108.

\section{REFERENCES}

1. Sethi NK, Mullin P, Torgovnick J, Capasso G. Nitrous oxide "whippit" abuse presenting with cobalamin responsive psychosis. J Med Toxicol 2006;2:71-74.

2. Eldibany MM, Caprini JA. Hyperhomocysteinemia and thrombosis: an overview. Arch Pathol Lab Med 2007;131:872-884.

3. Dong X, Ba F, Wang R, Zheng D. Imaging appearance of myelopathy secondary to nitrous oxide abuse: a case report and review of the literature. Int J Neurosci 2019;129:225-229.

4. Lan SY, Kuo CY, Chou CC, Kong SS, Hung PC, Tsai HY, et al. Recreational nitrous oxide abuse related subacute combined degeneration of the spinal cord in adolescents-a case series and literature review. Brain Dev 2019;41:428-435.

5. Kwoun YJ, Choi YJ, Oh JY, Park KD, Choi KG. Myeloneuropathy following chronic abuse of nitrous oxide. J Korean Neurol Assoc
2003;21:432-435.

6. Sun W, Liao JP, Hu Y, Zhang W, Ma J, Wang GF. Pulmonary embolism and deep vein thrombosis caused by nitrous oxide abuse: a case report. World J Clin Cases 2019;7:4057-4062.

7. Feng LY, Yu WJ, Chang WT, Han E, Chung H, Li JH. Comparison of illegal drug use pattern in Taiwan and Korea from 2006 to 2014. Subst Abuse Treat Prev Policy 2016;11:34.

8. American Association of Electrodiagnostic Medicine, Olney RK. Guidelines in electrodiagnostic medicine. Consensus criteria for the diagnosis of partial conduction block. Muscle Nerve Suppl 1999;8:S225-S229.

9. Choi C, Kim T, Park KD, Lim OK, Lee JK. Subacute combined degeneration caused by nitrous oxide intoxication: a report of two cases. Ann Rehabil Med 2019;43:530-534.

10. Lee HL, Lee SJ, Nam TS, Min SH, Kim JM, Kang KW. Acute cervical myelopathy following laughing gas abuse. Chonnam Med J 2019;55:118-119.

11. Richardson PG. Peripheral neuropathy following nitrous oxide abuse. Emerg Med Australas 2010;22:88-90.

12. Maler JM, Seifert W, Hüther G, Wiltfang J, Rüther E, Kornhuber J, et al. Homocysteine induces cell death of rat astrocytes in vitro. Neurosci Lett 2003;347:85-88.

13. Kölker S, Ahlemeyer B, Krieglstein J, Hoffmann GF. Methylmalonic acid induces excitotoxic neuronal damage in vitro. J Inherit Metab Dis 2000;23:355-358.

14. Weimann J. Toxicity of nitrous oxide. Best Pract Res Clin Anaesthesiol 2003;17:47-61.

15. Pleasure D. Toxic neuropathies. In: Darras BT, Jones HR, Ryan MM, De Vivo DC, eds. Neuromuscular disorders of infancy, childhood, adolescence: a clin approach. 2nd ed. Oxford: Elsevier, 2015:431437.

16. Tani J, Weng HY, Chen HJ, Chang TS, Sung JY, Lin CS. Elucidating unique axonal dysfunction between nitrous oxide abuse and Vitamin B12 deficiency. Front Neurol 2019;10:704.

17. Waclawik AJ, Luzzio CC, Juhasz-Pocsine K, Hamilton V. Myeloneuropathy from nitrous oxide abuse: unusually high methylmalonic acid and homocysteine levels. WMJ 2003;102:43-45.

18. Kwon YJ, Rho JH, Hwang J, Baek SH. Unhappy end of 'happy balloons': subacute combined degeneration caused by nitrous oxide gas. J Clin Neurol 2019;15:118-119.

19. Kang SW, Hong JM, Namgung DW, Choi Y. Neurological manifestations of myeloneuropathy in patients with nitrous oxide intoxication. J Clin Neurol 2019;15:116-117. 